\title{
THE ACUTE ABDOMEN
}

By H. W. S. Wright. M.S., F.R.C.s.

\section{"Our Natures are the Physicians of our Diseases."-Epidemics, vi. 5. \\ "Those Diseases that Medicines do not cure are cured by the Knife."-Aphorisms, vIr. 87.}

HIPPOCRATES.

The acute abdomen may be defined as an intra-abdominal lesion which, apart from appropriate treatment, immediately threatens the life of a patient. In England, with a population of nearly 42 millions, considerably more than 12,000 people die annually from what is called "an acute abdomen." The annual crude death-rate from appendicitis is 62 per million, and from hernia and intestinal obstruction rog per million. In greater London, with a population of nearly nine million persons, at least ro,00o per annum are admitted with a diagnosis which implies a major abdominal catastrophe. Because they are incomplete, these figures underestimate the magnitude of a problem which claims a large and important share of every surgeon's time and attention. It would be surprising if such a group of cases, with so much in common, did not have an embryological and biological background which deserves understanding and consideration.

When, in the course of biological evolution, the mesoderm became differentiated as a separate cell mass which later split to form a body cavity or coelom, the formation of this cavity marked a critical stage pregnant with possibilities. The organs inside the cavity were separated from the exterior for their specialised functions of digestion, absorption, and respiration. Such an advance removed many obstacles to increase both in size and variety, and determined the possibilities of coelomate forms. But the differentiation of an intestinal canal within a serous cavity implies the provision of a vascular system for its own nourishment and the transport of metabolites, a lymphatic system for absorption and protection, and a nervous and endocrine system for co-ordination and control. With this increased complexity there is necessarily an increased danger of breakdowns fraught with disastrous and far-reaching consequences. The existence of a coelom, in fact, implies the probability of the acute abdomen.

It is easy to see that organs, such as the appendix and the gallbladder, which are developed as blind diverticulae from the midgut, must always be liable to obstruction and subsequent infection, a series of changes so aptly called "the hollow viscus pathology." Inherent in the localising and protective functions of the peritoneum and omentum is the possibility of adhesions and obstruction, and the presence of lymphatic tissue thinly covered with muscle and peritoneum makes occasional swelling, necrosis, and perforation a certainty.
It is not proposed in this article to describe in detail abdominal conditions which are adequately dealt with in all standard textbooks, but rather to analyse their symptomatology and its mechanism in such a way that a clinical pattern emerges quite simply from a mosaic of apparently unrelated symptoms, and to show that the treatment suggested is a logical sequence to pathological findings.

The symptoms and signs which give evidence of an acute intra-abdominal lesion are as a rule few and simple. They are pain, superficial and deep tenderness, rigidity, and vomiting. With these are associated the general effects of the lesion on the whole organism, such as temperature changes, and alterations both absolute and relative, in the composition of the blood and urine. The integration of these signs and symptoms into familiar clinical patterns usually enables a diagnosis to be made with rapidity and certainty, often on the telephone; but in many cases the most meticulously careful history, precise examination, and the nicesto judgment are necessary before reliable conclusionso can be reached, and in order to do this some knowledge of the nature and mechanism of symptom production is necessary.

The pain of abdominal disease is of two kinds, visceral and somatic, and they can frequently be distinguished from each other by the description given by an intelligent patient, and sometimes by the patient's appearance. Visceral pain arises directly from pathological changes in the involved viscus or its mesentery and vascular connections, and somatic pain arises from the parietes as the result of secondary and coincidental changes. The classic example of visceral pain is that due to coronary thrombosis, to renal or intestinal colic. It is tearing, crushing, or bursting in quality, often severe enough to cause vomiting, rapid pulse, and some degree of collapse; the patient may roll about or double himself up in what he may well call agony. In the case of colic it rises to a crescendo and then diminishes, only to recur again. This type of pain is characteristic in that it can be abolished by section of the appropriate sympathetic pathways. It is usually imprecisely localised but has an area of reference which depends on the embryology of the implicated viscus. Very often the patient will say the pain is inside the abdomen. This type of pain is most easily distinguished at the onset of the attack before the adjacent parietal peritoneum is involved, and other protective reflexes are established. It is frequently but not 
invariably associated with rigidity and deep tenderness. For instance, the pain of renal colic is sometimes accompanied by rigidity and tenderness over an area wide enough to be suggestive of a perforation, whereas the pain of intestinal colic frequently is not, and if rigidity and tenderness are present, as a rule thev disappear soon after the colic ceases. The reason for the often vague localisation of visceral pain is that the afferent nerves from viscera traverse the ganglia of the autonomic system to the posterior nerve roots which are arranged segmentally, but the subjective aspect of localisation takes place in the brain where pain is registered in terms of quality and position. Neither of these latter attributes are constant, and vary from individual to individual.

For instance, the early pain in a typical attack of acute appendicitis is usually felt around the umbilicus. This might well be expected as the appendix is part of a medially developed midgut, but the pain is often described by the patient as arising in the epigastric region, and occasionally elsewhere. An analogy may perhaps make these individual differences comprehensible, if not reasonable. The quality of tones registered in a photograph varies with the composition of the film, so also do the spatial relationships vary with its contour. Seen in this way it does not seem unlikely that different individuals will describe the quality and position of their visceral sensations differently. That they do so is a fact of observation which has to be constantly borne in mind when assessing the value of a patient's statements.

The other type of pain which occurs in acute abdominal disease arises from inflammation of the neighbouring peritoneum, or reflex irritation of the overlying muscles. It is constant in position, unvarying in quality, and in the nature of burning or aching. When severe, it is difficult to distinguish from any other severe pain except that it is more precisely localised. It is nearly always associated with rigidity and deep tenderness. Since it arises from the parietes, the nerves of which pass through the appropriate posterior roots and retain their original segmental arrangement relatively undisturbed, localisation in the cerebral cortex is much more precise.

With both these types of pain is associated hyperaesthesia-pain which is superficial and has a stinging or itching quality. It is also characterised by the fact that it often persists for some long time after the original stimulus has ceased and may even be the last sign to disappear. Its mechanism is incompletely understood, but its characteristics suggest that it arises in the skin of the painful area. It is strictly segmental in distribution. Sir Thomas Lewis and his co-workers have shown that it may be produced by electrically stimulating the peri- pheral end of a novocaine-blocked sensory nerve. Since it arises some time after the stimuli, which probably do not reach the cord, have ceased, it is unlikely that it has its origin in the cord itself.*

Muscular rigidity is associated with both parietal and visceral pain. It may affect part of a muscle whose total nerve supply comes from several segmental nerves, thus producing a so-called phantom tumour, and it may persist for some days after the pain has ceased. This suggests that it may be due to a "facilitated reflex" and that a stimulus qualitatively below the threshold of pain may continue to produce rigidity when the pain has ceased. This persistence is not uncommon in inflammation of the gallbladder or appendix, and because of this, a surgeon on opening the abdomen, may sometimes find much less evidence of acute inflammation than he had previously led himself to expect.

The mechanism of deep tenderness is much more difficult to understand. It is as a rule coincident in time both with the pain and rigidity. It probably arises in the muscle itself because, as Lewis has shown experimentally, muscle is tender after contracting for two or three minutes, and after spasm of longer duration muscular tenderness may persist for some time. This tenderness is probably related to partial ischaemia, but whatever its cause everyone is familiar with the stiffness and tenderness which follow prolonged unaccustomed exercise. It is however certain that ischaemia does not represent the whole story, since deep tenderness in the testicle may be produced either by renal colic or by the intraligamentary injection of saline.

Pain, rigidity, deep tenderness, and hyperaesthesia then are the cardinal symptoms of acute abdominal pathology. It is wise to remember that the severer types of visceral and somatic pain may be indistinguishable, and that either or both together are often associated with their reflex effects, rigidity, deep tenderness, and hyperaesthesia. These last three may also be caused by inflammation in the muscles and ligaments of the appropriate segments and can be exactly reproduced by the intra-ligamentary injection of saline. (Lewis and Kelgren). Obviously, it would be unwise to assume that an understanding of the mechanism of symptom production is unimportant for the diagnosis of acute abdominal disease. Every casualty officer is aware that the diagnosis is sometimes made correctly by a parent or a policeman, but every surgeon has on his conscience a death or weeks of hospitalisation which might have been avoided had an obscure case been more carefully considered.

* For a full discussion of this question the reader is referred to ch. xiii of Sir Thomas Lewis's book on Pain. 
Acute abdomens may, for descriptive purposes, be divided into four groups which, of course, necessarily overlap.

(I) The obstructive visceropathy of which appendicitis and cholecystitis are examples.

(2) Perforations of ulcers or inflamed and gangrenous viscera.

(3) Obstructions with or without interference to the vascular supply.

(4) Vascular obstructions due to intravascular, or extravascular mechanical causes, such as mesenteric thrombosis or the torsion of an ovarian pedicle. An analysis of 348 cases admitted to an emergency hospital which served a large semiurban and rural area, shows that these types occurred in the following proportions:

Obstructive visceropathys $6 \mathbf{I} \cdot 5 \cdot$ per cent; Perforations 9.5 per cent; Obstructions 25.3 per cent; Vascular obstructions due to Torsions 3.7 per cent.

\section{(I) The Obstructive Visceropathy}

Acute appendicitis.-Though most cases occur in adolescence and early adult life, it may occur at all ages. It is particularly dangerous and often difficult to diagnose in childhood and old age. Moreover, the appendix is subject to such great variation in position that its manifestations are apt to be anomalous. It is therefore wise to remember in obscure cases that. one is always more likely to encounter an unusual variant of a common disease than a rare disease. Its onset is usually sudden and begins with colicky or cramping pain referred to the midline near the umbilicus. This pain is accompanied by vomiting in many cases. It can be reproduced by pulling on or squeezing the appendix when the abdomen is opened under local anaesthesia. If the patient is examined at this stage there will generally be deep tenderness over McBurney's point. A little later there is pain, more or less severe and constant, associated with the défense musculaire of the French writers. This consists of rigidity, deep and superficial tenderness over an area corresponding to the position of the appendix, usually the right lower quadrant of the abdomen. By this time the vomiting has ceased, the patient may seem quite well, the temperature normal or only slightly raised, and the pulse hardly raised at all. The exact physical signs vary with the position of the appendix; a retrocaecal appendix may give little rigidity or pain in the early stages, a pelvic appendix often almost none, but here rectal or vaginal examination will help. When the appendix is situated lateral to the caecum a localised mass may be felt or there is rigidity in the loin, and when it is surrounded by omentum a mass may be palpable near, or beneath the edge of the right rectus. Such a mass may be so protected by omentum that it is not very tender and even allows a limited mobility on palpation. An appendix situated so that its tip rests on the psoas will give rise to spasm of this muscle, flexion of the thigh, and pain, when the latter is extended.

The most difficult situation arises when the appendix is in the pelvis. The area of peritoneum within the true pelvis is a silent area and gives rise to little rigidity, abdominal pain, or hyperaesthesia. The disturbances of defaecation and micturition so often mentioned in textbooks are more often absent than not, but when the condition is well developed there is usually, tenderness per rectum or per vagina.

One type of obstructive appendicitis, specially $\frac{8}{0}$ emphasised by the late Sir David Wilkie, requires 3 . special mention. There occurs an acute obstruc- iv tion of an infected appendix, and a virulent $\stackrel{N}{\sim}$ organism causes inflammation and early gangrene. $\stackrel{+}{\infty}$ Often there is early vascular thrombosis. The $\vec{A}$ onset is very sudden and the pain is very severe, $\overrightarrow{0}$ colicky in type, and referred as a rule to the 9 umbilicus. The patient is doubled up and vomits with each spasm. $\mathrm{He}$ is often collapsed and $\zeta$ anxious looking during and after the attack of $\underset{\varpi}{\mp}$ pain. At the onset there is little to show in $\vec{\theta}$ between the spasms; there may be a little tendernes deep in the right iliac fossa and some hyperaesthesio

To an experienced observer the patient gives these impression of having suffered a great deal of paint, and often seems a little more "knocked" than he o would be if it were mere intestinal colic. In this $\stackrel{\mathscr{\partial}}{\partial}$ type of case every hour that passes is of importance, $\stackrel{\varnothing}{\unrhd}$ forperforation and general peritonitismay occur early $\overrightarrow{\vec{D}}$ without preliminary signs of local peritonitis. If 3 the doctor who is responsible for such a case is in doubt he may well remember Moynihan's dictum that every abdominal pain which requires more than $\frac{0}{0}$ one dose of morphia for its relief, probably requires $\underline{3}$. laparotomy for its cure; or Zachary Cope's advice that if pain, assumed to be due to intestinal colic, $\stackrel{3}{.}$. persists for more than three or four hours, $ᄋ$ the condition calls for surgical interference.

Gentle palpation starting from the silent to the $\frac{9}{9}$ symptomatic side of the abdomen will generally detect some guarding and rigidity. If the pressure of the hand on the normal side is suddenly released there will be pain over the area of any inflamed o viscus, and this is often valuable evidence of early $\tilde{0}$ localised peritonitis. A tentative diagnosis can $\underset{\omega}{\mathcal{W}}$ often be made by watching the abdomen closely $\mathrm{O}$ in a good light; a localised area of muscular rigidity 0 can then be seen as the rest of the abdomen moves ${ }_{\mathbb{D}}$ with respiration, and over such an area the $\stackrel{\oplus}{\sim}$ abdominal reflex is almost invariably absent.

There is nearly always a slight rise in the $\overrightarrow{0}$ leucocyte count or a "shift to the left" in the 
Arneth count. These changes may be of importance in distinguishing appendicitis from other extra peritoneal conditions causing localised pain and rigidity. The total white count is rarely more than I2-I4,000, and it is of more prognostic than diagnostic importance.

These clinical findings represent, in terms of gross pathology, inflammation of the appendix and the adjacent peritoneum.

From this stage onwards three things may happen. The whole process may, and often does, completely subside, leaving increased liability to a future attack. But the appendix is largely composed of lymphatic tissue, and if it is obstructed, as it nearly always is, either by its mesenteric attachments or by a faecolith, it may necrose and perforate. Such cases will gradually develop first a localised, and later a general peritonitis. Sudden perforation is often the signal for the temporary cessation of the symptoms, but soon the pulse rate rises, rigidity and pain increase, the temperature drops, or occasionally rises according to the patient's resistance, and vomiting and other toxic symptoms supervene. Finally, the whole process may become localised and an abscess or inflammatory mass be formed. If this happens, as the general rigidity diminishes a mass becomes differentiated; the temperature and white count behave as they do in any other abscess.

The treatment of acute appendicitis is by now standardised, it is operative; but this does not mean that it is unnecessary to exercise wise judgment and careful consideration. It is not very helpful to say that the surgeon should hold his hand after the first 24 or 48 hours, because cases develop at very different rates. Perhaps the indications for conservative treatment are best described by saying that no wise man would either interfere with a localising peritonitis, or disturb an inflammatory mass where there were no indications that it contained pus.

In principle the appendix should be removed by an incision as far from the midline as possible when this can be done without unduly breaking down adhesions or spreading the infection. The abdomen should be drained if there is pus, an oozing surface, or necrotic material left behind. In a few suitable borderline cases it is justifiable to use a spinal anaesthetic because the "quiet" abdomen it gives may enable an appendix to be safely removed when removal would otherwise be dangerous.

If an appendix containing pus is attached to the posterior abdominal wall, the protective barrier on its posterior surface should in no way be disturbed, since this may result in a retroperitoneal cellulitis, a condition of affairs which is frequently fatal. The area should be carefully surrounded by packs, and the base of the appendix divided and buried. It should then be detached from above downwards to where it is adherent to the posterior abdominal wall, and then the lumen may be opened, the pus wiped away and the necrotic mucosa gently curetted and the raw surface cauterized. Sometimes it is possible to divide the muscle down to the mucosa and remove unbroken a tube of mucosa containing pus. Both these procedures are occasionally life-saving. (Figs. I and 2.)

It is still important to emphasise that in general the treatment of acute appendicitis is immediate operation. A few hours in bed to get the patient rested and quiet is often judicious. A doubtful diagnosis in the patient's house or casualty room can often be cleared up in this way, but if the diagnosis is still doubtful and appendicitis probable, it is wise to look and see. Some years ago a very distinguished surgeon in a presidential address to the Royal Society of Medicine rightly emphasised how often acute appendicitis settled down and resolved. This perfectly proper statement to a medical audience unfortunately got into the lay Press, and in the succeeding three months the writer of this article saw two deaths which were the direct result of this statement. In the writer's experience of 200 cases diagnosed as appendicitis, every doubtful case was operated on, two unnecessary laparotomies and appendicectomies were however performed. In another nearly paralleko series in which operation was delayed until the? diagnosis was absolutely certain there occurred one sub-diaphragmatic abscess, and another case which had a subdiaphragmatic abscess and an abscess of the lung which required drainage and later a plastic procedure. There is one sinister fact which emerges from any series of cases of acute appendicitis, and that is that no one can say what the outcome will be. The operation findings are frequently at variance with what one would expect. This is counterbalanced by the incontrovertible truth that operations performed while the disease is limited to the appendix and its immediate surroundings carry a very low mortality, little morbidity, and a rapid convalescence.

During the period I943-1945 in which the figures previously mentioned were collected, five normal appendices were removed as the result of an erroneous diagnosis. In twelve cases the abdomen was opened because appendicitis was diagnosed, and other conditions requiring operation were found, and in ten cases other conditions were diagnosed when the patient actually had acute appendicitis. In four cases the only pre-operative diagnosis was general peritonitis.

It is also interesting to note that of the I94 cases of proved acute appendicitis, 50 were drained, and in $\mathrm{I}_{4}$ of these fifty, the appendix was not removed. 
Twenty-three of the fifty drained cases occurred in the "flying bomb period" when it was noticeable that patients tended to arrive at a later stage than the average.

Acute cholecystitis bears close comparison with acute appendicitis, for the gall bladder is a hollow viscus with a narrow, tortuous, and easily obstructed outlet. It is, however, a muscular organ connected with a normally sterile biliary tract, and so is less likely to be acutely inflamed than an organ mainly composed of lymphatic tissue draining directly into what may be regarded as a septic tank.

For purposes of description cases may be conveniently divided into those associated with gallstones and those without, but this classification is not so convenient clinically as it appears on paper, because an acutely inflamed gallbladder which is obstructed by a gallstone does not always cause biliary colic, and conversely, a gallbladder obstructed by oedema or a plug of mucus in the cystic duct sometimes is associated with colicky pains at the onset. It is, moreover, frequently stated that an acute cholecystitis without gallstones is the result of a general infectious disease such as typhoid. This is by no means always true.

However difficult it may be accurately to integrate the clinical with the pathological findings in any given case, it is always clear, as in appendicitis, that obstruction of some kind or other determines the onset of acute suppurative or gangrenous cholecystitis. The operation findings make this so obvious that the bacteriology is apt to receive insufficient attention.

The infection is usually mixed, the bacillus coli predominating but associated with a streptococcus. In the severer cases, particularly those associated with marked toxaemia and a rapid progress, the dominant factor is often the presence of anaerobic gas-forming organisms. (Gordon Taylor and Whitby. B.J.S. I930, xviii, 38.)

Clinically the patients often (6o-70 per cent) give a history of flatulent dyspepsia suggestive of cholelithiasis, and gallstone colic, more or less severe, initiates the attack. The pain is sudden in onset, situated in the right hypochondrium, and radiating outwards to the axilla or across the abdomen. It doubles the patient up and may cause vomiting. Hyperaesthesia and deep tenderness which persist after the colic has passed off, are present at a spot just medial to and below the angle of the scapula. Instead of passing off as colic usually does, it is succeeded by pain, soreness and rigidity in the right upper quadrant of the abdomen, which is due to local peritonitis, and if the diaphragm is involved, pain is referred to the tip of the shoulder. The rigidity may extend all down the right side but is at its maximum above.
Horace, who lived in an age of gastronomic indulgence, was familiar with the referred pain of gallstones, for in describing an attack of colic which Maecenas suffered, he mentions the burning in the shoulder.*

The physical signs may be difficult to differentiate from those due to laterocaecal appendicitis until the abdomen is relaxed under an anaesthetic, when a characteristic mass may be palpable. The temperature is higher than in appendicitis $\left(\mathrm{IOI}^{\circ}-\mathrm{IO}^{\circ} \mathrm{F}\right.$.) as is also the white count. There is frequently some congestion at the base of the right lung. The patient very often seems ill and toxic.

The course of the disease from this point onwards varies. It has to be conceded that by far the greater majority of the cases settle down. The rigidity passes off, leaving a palpable mass which is usually omentum attached to the gallbladder, but sometimes a large pyriform, distended gallbladder can be felt. This occurs when a cholesterin stone is impacted in the neck of the gallbladder and has previously caused a mucocoel.

In other cases signs of toxic absorption may increase and the process go on to general peritonitis; or some early localisation and abscess formation may take place, to be succeeded later by widespread generalisation. There is a frequently expressed difference of opinion between experienced surgeons about the treatment of acute cholecystitis. Some maintain that, like acute appendicitis, the outcome is uncertain and it should be operated upon at once, ? since operation in the first $24-48$ hours is easy, and little different from the inevitable operation later on. Against this is the fact that one seldom sees cases so early, and that the majority of them do settle down. There is no type of acute abdomen which requires more careful individual consideration, and probably opinions which seem to differ fundamentally when expressed in writing, would agree more closely when applied to $\cdot a n$ actual case. There would be general agreement upon the following points:

(I) That with few exceptions, every case may safely be given a few hours of rest in bed for adequate preparation, in particular the administration of fluid and glucose and one dose of morphia and atropine.

(2) That a case seen in the first 24 hours and before the succeeding inflammatory reaction is marked, can be safely operated upon early since adhesions are oedematous and strip easily.

(3) That after this early period has passed, the case may be carefully watched to see whether or

* Nec munus humeris efficacis Herculis Inarsit aestuosius. (Epodes iii, I7, I8.)

Nor did Nessius' gift burn with fiercer flame into the shoulder of Hercules. (Gordon Taylor. B.J.S., I937, XXv, I6.) 
not the temperature and particularly the pulse rate diminish, and that any steady increase in the pulse rate is an indication for immediate interference.

(4) That a total and differential leucocyte count should be done at once, not so much for its immediate value as for comparison later.

(5) That if, after rest and glucose, the patient still looks toxic and the pulse is raised out of proportion to the rest of the physical signs, operation should be immediate in view of the possibility of an anaerobic infection.

(6) That if it is decided to allow the case to settle down, it should be most carefully watched, and that, although they may cause pain, breathing exercises should be instituted, and sulphonamides and penicillin should be administered.

The ideal treatment for acute cholecystitis is cholecystectomy, but this is often more easily said than done, and beyond peradventure it is no operation for an inexperienced surgeon. Apart from gangrene, it should not be done unless the cystic duct and the common bile duct are completely exposed, a procedure which may be timeconsuming, difficult, and even impossible. As a rule the common bile duct should be explored, a $\mathrm{T}$ tube put in, and after removing the gallbladder, the whole region adequately drained through a separate stab wound. Failing this, the gallbladder should be aspirated, the obstructing stone removed, and then simply drained, the tube being passed first through a hole in the omentum and then through a stab wound in the abdominal wall at a point corresponding to the lower border of the liver. In the writer's experience with a proper selection of cases, this has proved rapid, simple, and safe, whereas, although cholecystectomy has been technically successful, seemingly satisfactory, and associated with a low mortality, the morbidity and complication rate of cholecystectomy in severe cases has seemed unduly high. Furthermore, the secondary cholecystectomy done in a few months' time has invariably been easier than one would expect, and the convalescence rapid and smooth. It has in fact been smoother than after cholecystectomy done a fortnight or three weeks after the acute attack has subsided. This latter is what many surgeons tend to do when the condition is obviously subsiding:

If there is a patch of gangrene present on the fundus, it is always possible, after aspiration and adequate packing, to divide the gallbladder below this and to cut away its walls up to where they are attached to the liver. The remainder is then thoroughly coagulated with a diathermy button. A drainage tube is tied into the stump and the whole covered with omentum. Even if a complete formal cholecystectomy can be done, the part of the gallbladder attached to the liver may well be left behind and lightly coagulated, for it is under $\frac{0}{\pi}$ these circumstances that Thorck's technique best 0 serves its purpose.

Six of the twelve cases in this series had a $\stackrel{\varnothing}{\circ}$ cholecystectomy, and in six the gallbladder was $c$ drained.

In these acute cases anaesthesia is supremely $\frac{5}{0}$ important; probably gas, oxygen and cyclopropane, 듬 with or without local block, is the ideal, but it $\overline{\frac{F}{n}}$ needs a skilled and experienced anaesthetist. Fail- $\widehat{\varnothing}$ ing this, careful upper abdominal field block combined with gas, oxygen and a little ether is $\%$ satisfactory and very safe.

\section{Acute Perforative Diverticulitis}

Another example of the same type of pathological $\frac{8}{3}$ process is seen when a diverticulum of the left side of the colon perforates. Acute perforative diverti- $N$ culitis must be distinguished from a moregeneralised, $\stackrel{ \pm}{ \pm}$ less acute inflammation which sometimes flares up in a segment of bowel already the site of chronic $\vec{b}$ diverticulitis. In the latter case there is a general $\circ$ inflammation of the whole mass accompanied by a localised peritonitis. The whole process is rather $c$ similar to acute appendicitis, but the constitutional $\subsetneq$ symptoms tend to be more severe: There may, for ${ }^{\Phi}$ instance, be a rigor, and the patient looks ill. Thig $\vec{\oplus}$ is preceded by an exacerbation of the previou rather mild discomfort; for example, some increasec difficulty in defaecation, and colic referred to the left side of the large bowel. It should be treated conservatively with absolute rest, and until it subsides, only enough fluid and glucose given by $\frac{0}{\circ}$ mouth to prevent thirst.

In contrast, the perforation of an inflamed diverticulum precipitates a dangerous condition which cannot but give rise to great anxiety. It is strictly comparable to the perforation of an inflamed appendix. The patient, often obese, is as $\frac{0}{3}$ a rule too ill to give a detailed history which would reveal recent colic, localised pain, tenderness, and 3 constipations against a background of previous diverticulitis. The temperature at first is high 3 $\left(\mathrm{IOO}-\mathrm{IO} 4^{\circ} \mathrm{F}\right.$.) and there are the physical signs of a rapidly spreading. peritonitis which has its origin in the left iliac fossa. Rigidity, which the gentlest $\frac{}{0}$ and most persuasive palpation fails to overcome, hides the characteristic tumour lying parallel to 0 Poupart's ligament. If untreated and unrecog- $N$ nised the inevitable consequence is general peritonitis, the terminal phases of which was vividly described by Hippocrates and even recognised by Shakespeare-_I saw him fumble with the sheets. $\therefore$ For his nose was as sharp as a pen and a' babbled of green fields."

Operation should be immediate and a plasma or $\frac{T}{0}$ glucose drip transfusion is advisable. When the $\underset{\oplus}{\stackrel{0}{0}}$ 
abdomen is relaxed the tumour can be felt, and this gives the clue both to the diagnosis and to an appropriate incision, which should preferably be oblique, splitting or dividing the internal oblique and transversalis muscles. The pelvis and left side of the abdominal cavity is found full of evil, foul-smelling pus, but the actual perforation is seldom seen. The involved bowel should be wrapped with omentum, or, if this is impossible, covered with a free omental graft. As much as possible of the pus should be aspirated and the pelvis drained through a suprapubic stab wound. Five to ten grammes of a sulphonilamide powder may then properly be put in the peritoneal cavity, though perhaps it is better added to an intravenous drip. The wound should be sewn up with single catgut stitches, leaving a drain at the lower end which reaches the left iliac fossa and the pelvis.

However desperate the state of the patient, under no circumstances should he or she be sent back to bed without some form of right-sided transverse colostomy designed completely to divert the faecal stream from the sigmoid. If time presses and anxiety for the patient's life dominates the situation, a large suprapubic drain through a small incision combined with a quick transverse colostomy over a glass rod are good tactics and may save a desperate situation. From then on till the tide of battle begins to turn, the most useful weapons are morphia and prostigmine, a Ryle's tube and an intravenous drip, each used with discretion and judgment.

Another condition which falls naturally into this group and clinically very much resembles the last, is an acute suppurative salpingitis. This arises in an old and cold bilateral salpingitis of gonococcal or puerperal origin. From the bowel or blood stream it gets reinfected with a virulent colon bacillus which is sometimes accompanied by a streptococcus. The infection, after a brief and relatively silent sojourn in the pelvis, spreads rapidly and virulently to the lower abdomen. In the early stages one side or other bears the brunt of the attack, and it is by general surgeons often mistaken for a perforated pelvic appendicitis or an acute perforative diverticulitis. As far as the abdominal cavity is concerned, the peritonitis has an abrupt onset, and, unlike the conditions which it mimics, it is unheralded by symptoms referred to the abdominal viscera, but a long-standing dysmenorrhea and some symptoms referable to micturition or defaecation may obtrude themselves into the clinical picture. It has, however, some invariable characteristics indicating its origin. There is always a previous vaginal discharge and a bilateral tender mass in the fornices which corresponds to the infected obstructed tubes. These are not, however, quite always palpable, for in a recent case, diagnosed as diverticulitis, they were taken out of reach and covered by a large fibroid, previously known to be present.

The diagnosis is often missed because the condition is not well recognised by general surgeons. Out of seven such cases, four were thought to be acute appendicitis, one a leaking ectopic gestation, and in two no exact diagnosis was made.

There is usually no doubt that the abdomen ought to be opened, and as the condition is bilateral a median or paramedian subumbilical incision should be used, in fact these cases are almost unique in that they are examples of lower abdominal infection which it is wise to approach from the middle line. When the abdomen is opened no attempt should be made to remove the tubes, loculi in front of and behind the broad ligament should be gently opened and the pelvis drained. Many gynaecologists would prefer a drain inserted through the posterior vaginal fornix. The prognosis is good if the operation is promptly and rapidly carried out.

\section{(II) Perforations}

The perforation of a gastric or duodenal ulcer is one of the most serious and imperative of all surgical emergencies, but compared with many others it is easy to diagnose and simple to treat Like the entirely mythical Celt in Kipling's poem "One knows what he will do, and you can logically predicate his finish by his start." Most perforated ulcers are situated on the anterior wall of the pyloric antrum or duodenum. Ulcers on the lesser curve or posterior wall are more likely to penetrate the pancreas or liver. Most of them are chronic ulcers in which activity has recrudesced by a rapidly spreading infection beneath the callus edge. The condition has been described by Bolton as the acute spreading ulcer. Occasionally acute ulcers may perforate, especially when they are embolic in origin, the primary infection being a burn or a septicaemia.

The base of a perforated ulcer is usually rigid and friable and shows signs of recent inflammation. This is reflected in the immediate history. As Moynihan has pointed out, an ulcer very rarely perforates without giving some warning of the impending catastrophe. Pain increases and tenderness is present although the patient may be too ill to mention it. That perforation may occur while the patient is under treatment is not sufficiently well known, and more than one has been "cooked" for twelve hours because the doctor or the house physician thought that an ulcer under a strict medical regime could not possibly perforate.

Everyone is familiar with the sudden onset of a perforated ulcer. The resulting acute epigastric 
pain is associated with boardlike upper abdominal rigidity which then spreads downwards, more to the right than theleft, and gradually becomesgeneralised. The pain is constant, widespread, and very severe, differing from colic in its unvarying intensity and the way it makes the patient hold himself quite still. It is noticeable that respiration is almost entirely intercostal, and although the rigidity is widespread, it reaches its maximum in the upper abdomen. Such patients also only vomit once or twice in the early stages. What is not so often realised and needs emphasis is, that the temperature is seldom raised, or even is a little sub-normal, and that the pulse rate is frequently normal or only slightly raised, though the volume may be a little increased. These patients occasionally walk into hospital. Only later does the pulse rate increase and collapse supervene. Notwithstanding all this, to an experienced eye, the patient nearly always gives the impression of having suffered a good deal of pain. General toxaemia comes relatively late and rigidity passes off as the irritation of the concentrated gastric contents subsides owing to dilution with inflammatory exudate. At the beginning of this stage the patient may even seem a little better. Then the temperature rises, he is flushed, and the pulse rate increases. Laterstill, pain, rigidity and tenderness decrease as the long grey shadow of collapse and toxaemia due to general peritonitis slowly obliterates the outlines of a clearcut clinical silhouette.

One difficulty in diagnosis often arises. A perforated duodenal ulcer may discharge its contents down the right paracolic gutter and they willaccumulate first in the right iliac fossa. As a result the défense musculaire reaches its maximum here and acute appendicitis is clearly mimicked. But nearly always, in the history, the symptoms of a duodenal ulcer can be discerned building up to a sudden acute epigastric pain which leaves in its wake some definite rigidity and tenderness over the upper part of the right rectus muscle.

Of ancillary methods of diagnosis the most useful is a plain X-ray which may show a bubble of gas beneath the diaphragm. This is more reliable than obliteration of the liver dullness, but neither of these findings is by any means constant.

Soon after the patient is admitted it is wise to pass a Ryle's tube and by frequent aspiration keep the stomach empty. If the patient is collapsed a continuous plasma drip should be put up, the first bottle being run in rather rapidly, for these patients lose a lot of protein in the voluminous peritoneal exudate which is literally poured out: When on account of venous collapse the transfusion will not run well, an occasional case may be saved by using two veins at the same time. Operation should be early and expeditious. In no type of abdominal emergency is delay more dangerous and it should never await the convenience of the $\frac{\varrho}{z}$ surgical team for more than an hour or two. The $\frac{\mathbb{Q}}{2}$ shortness of the time that elapses between per- $\frac{\varrho}{c}$ foration and operation overshadows in importance every other prognostic factor. This statement $\stackrel{\vec{S}}{+}$ perhaps has one exception of practical importance. 을 The outlook is bad if the patient has had a recent $\frac{\bar{\sigma}}{\bar{N}}$ haematemesis, and the technique of closure should $\frac{\overline{\bar{m}}}{\overrightarrow{0}}$ be adapted to prevent its recurrence. Blood in the $\underset{\varrho}{\mathbb{Q}}$ transfusion may then be indicated as well as plasma.

The following figures serve to emphasise these $\overrightarrow{ }$ facts. Of twenty-two perforated ulcers of the? stomach or duodenum, seven died. One of these $\vec{\omega}$ had a carcinoma, one was first seen eighteen hours $\stackrel{\omega}{\circ}$ after perforation, and two had had a recent haema- 0 temesis. Of the remaining three, one had a sub- 3 . phrenic abscess, and two were too ill for any N interference whatever. The first of these had a $\mathrm{N}$ concomitant coronary thrombosis and the second $+\frac{\infty}{+}$ had perforated three days before admission.

As far as anaesthesia is concerned, perhaps it is $\overrightarrow{0}$ here that cyclopropane or curari will easily come을 into their own. They give rapid and immediaterelaxation without anoxaemia. Local anaesthesia can be used, but painful impulses come from such a wide area that relaxation is difficult to obtain, but $\vec{c}$ combined with gas, oxygen and ether, it is vegy satisfactory. Except in specially selected cass; spinal anaesthesia, with all its obvious advantag too often increases the percentage of respiratorys complications.

The operative technique should be as simple aső possible. The essentials are a right paramedian@ incision, three widely placed catgut stitches, one $\overrightarrow{\overrightarrow{0}}$ just beyond each end of the friable ulcer base,, 3 and one in the middle. These are tied, not too? tightly, over a thick piece of omentum, which: may if necessary be doubled. Then aspirate the right kidney pouch and mop up any obvious soiling Put a tube in the pelvis in most cases, and close the peritoneum. When this has been done spend? any time there is to spare in carefully cleansingo and sewing up the abdominal wall.

At this juncture a word should be said abouto those cases of obvious general peritonitis which are admitted to hospital so ill that a history isㅡ. unobtainable, and with such widespread rigidityn that reliable assessment of its origin is impossible? Under these circumstances the only policy for as surgeon to pursue is to emulate the bookmakersw and back the most probable winner. Appendicitiș is by far the most common cause of generab peritonitis. Therefore a short incision should be्ष made either over the appendix or in the midline just above the pubis. If the pus is foul ando stinking it probably comes from the appendix $\overrightarrow{0}$ If it is thin, slimy, and contains particles of food? 
it is probably due to a perforated ulcer. In either case the pelvis should be drained. It never does any harm to make a short incision in the wrong place, but much harm is frequently done by an attempt at exploration in the presence of sepsis through a median subumbilical incision.

Another type of acute abdomen which falls a little uneasily within the category of a perforation is a ruptured ectopic gestation. This is popularly supposed to produce a very clear-cut and characteristic clinical picture. The patient misses a period and a few weeks afterwards bleeds more profusely and longer than she would expect from her previous experience of menstruation. About the same time she is seized with severe abdominal pain, and on examination she has the other signs of peritoneal irritation. The diagnosis is clinched by the discovery of a mass in one or other vaginal fornix. My colleague, Mr. R. L. Dodds, has recently pointed out (Proceedings of the Roy. Soc. $M e d$. ., Vol. XXXIII, No. I2) that this is in fact a film version of a not very common tragedy, and its widespread acceptance results in the diagnosis being made incorrectly in about 70 per cent of all the cases. There are three good reasons for this otherwise deplorable state of affairs. Firstly, in the practice of a general physician or surgeon the condition is distinctly rare. Secondly, the differential diagnosis is often really difficult, and thirdly, as has already been pointed out, the facts that emerge from an analysis of the clinical findings are at variance with the average conception of the disease. The missed period, usually regarded as the key to the clinical puzzle, cannot be established in just under half the cases. In more than a third of them it is masked by the fact that the bleeding occurs just when the next period is due. Only a wary cross-examination will elicit the fact that the period just before the pain came on was more profuse and more prolonged than usual. Very occasionally this pseudo-menstruation appears a week early. The pain too is without distinctive features. It is felt typically in the lower abdomen, often more on one side than another, and when it appears it remains constant for some time; then there may be an interval of relative freedom. This must be distinguished from colic, which reaches its maximum quickly and then fades with equal rapidity. Sometimes the pain is generalised, occasionally it is referred to the epigastrium. There is always tenderness, and as often as not more rigidity than the intensity of the pain would seem to warrant, but cases are seen with a lower abdominal wall which reacts to and resists the slightest intrusion of an examining hand. Unlike appendicitis, this abdominal pain is never preceded by peri-umbilical colic and vomiting. Shoulder pain, sometimes quite severe, occurs in about one-third of all the cases, and if the lower abdomen is suspect, gives away the diagnosis- "An acute appendix with shoulder pain is a ruptured ectopic."

On pelvic examination one expects to find generalised tenderness or a tender mass in one or other fornix, but this is also found with acute salpingitis. It is also characteristic that movement of the cervix in an anterio-posterior direction causes a sudden sharp pain which makes the patient stiffen and catch her breath.

Acute appendicitis, acute salpingitis, and an early abortion are the three conditions which confuse the issue. The differences between ruptured ectopic gestation and acute appendicitis have already been indicated. A mistake is not important and its worst consequence is a short incision in the wrong place.

When acute salpingitis is confused with a subacute ruptured ectopic, three or four days' observation will settle the question; the salpingitis will invariably subside.

Examination under anaesthesia may be necessary to differentiate an early abortion. Tenderness and resistance is abolished and it is usually clear whether the uterus or a Fallopian tube is the seat of the pregnancy.

Little needs to be said about treatment once the diagnosis is made. The abdomen should be opene in the middle line and the involved tube removedo A blood transfusion is often wise and sometimes necessary. If strict antiseptic precautions are taken the patient's own blood may be used for replacement.

\section{(III) Obstructions}

Intestinal obstruction is by no means the most frequent cause of an acute abdomen, but it has a higher percentage mortality than any of the other types ordinarily met with in surgical practice. That a large part of this mortality is preventable because it is due to delay in diagnosis will readily be conceded by anyone who has to deal with many cases.

There is a legend that elaborate classifications of acute intestinal obstruction are much beloved of examiners, and most surgical coaches have their own favourite table of causes. These may indeed form a useful framework for an examination paper, but they are not of much practical clinical importance. When a clinician is confronted with a probable case, these are the intensely practical questions he has to ask himself:

(I) Is obstruction present or not?

(2) If so, is it in the colon or the small intestine?

(3) Does it, or does it not threaten the integrity of the bowel wall, or in other words, is it accompanied by vascular obstruction? 
It is not possible to over-emphasise the fact that an answer to these questions is possible in nearly every case by simple clinical examination assisted only by a plain X-ray of the abdomen, which may well be taken between the casualty-room and the ward. Now intestinal obstruction produces four cardinal symptoms, pain, vomiting, distension and sometimes tenderness, symptoms which are common to every other type of acute abdomen. It is therefore obvious that their significance lies in their mutual relationships.

Pain.-The pain is due to intestinal colic. It is intermittent, cramping, reaches its maximum quickly and retains its intensity only for three or four minutes. Its onset and departure are both equally abrupt. The colic due to intestinal obstruction is always accompanied by bubbling and gurgling which can be heard and often felt as the pain reaches its maximum intensity. Except in pyloric stenosis and obstruction at the efferent loop of a gastrojejunal stroma, this relationship is invariable and generally establishes the presence of obstruction of some kind.

Vomiting.-In all obstructions of the small intestine vomiting is present. It is frequently copious because it comes from the regurgitation of the intestinal contents into the stomach. The higher the obstruction the earlier it appears and the less likely it is to be faeculant. It gives only temporary relief or no relief at all to the patient's distress since more fluid from below is again regurgitated into the stomach. This vomiting tends to recur soon after a spasm of pain.

The vomiting of pyloric stenosis relieves the patient for the time being and, except in the very early stages before the stomach is dilated, is painless. The vomiting due to biliary or renal colic is not copious, is very exhausting, and is more in the nature of continuous retching which brings no alleviation of the patient's suffering. For reasons which will be discussed later most patients with obstruction of the colon do not vomit.

Distension.-Distension is visible or palpable in most cases and can always be demonstrated by a properly taken plain X-ray of the abdomen. Gentle palpation with a warm hand may detect the smooth, tense outline of a coil of small bowel, or general distension may be obvious.

The "ladder pattern" illustrated in textbooks is so late a manifestation that it has little real clinical interest. Visible peristalsis, which may be seen in thin abdomens, apart from obstruction, is of immense diagnostic importance when it is associated with colic.

In obstruction of the large intestine the colon is always distended and can usually be palpated. Even with obstruction of the distal colon the caecum is the first to dilate, and in the early stages colicky pain referred to a distended caecum may be the presenting symptom. In nearly all cases of large intestinal obstruction the ileocaecal valve is competent, so that vomiting is absent and the small intestine is not distended. The colic is referred to the segment of the colon proximal to the obstruction, and the distended bowel may be seen to contract, but this colic is not so prolonged as when the small intestine is involved, as the relatively short colon distends rapidly, is soon paralysed, and so cannot contract.

Occasionally, however, the ileocaecal valve is incompetent, and then the whole intestinal tract becomes involved in the resulting distension. A plain X-ray, taken in the supine position, will show the outline of a dilated colon down to the obstruction. The jejunum is recognised by its "feathery" appearance and the numerous cross striations. In contrast, the ilium is thinner, less "interesting," and has fewer cross markings. Fluid levels may also be seen, especially if it is possible to take a film in the upright position. (Figs. 3,4 and 5.)

Rigidity and guarding make it clear that transudation is taking place through the bowel wall, and, since this fluid is very irritating, it may even cause shoulder pain. There is an occasional but important exception to this last statement. A typical inflammatory lesion causing simple obstruce tion may cause localised tenderness and rigidityo The distinction can as a rule be made by relating the history to the physical signs, although it has to be admitted that this may sometimes be difficult or even impossible. It is, however, none the less important, because hasty interference in such cases usually results in spreading the infection, and not infrequently in the death of the patient. Suction, with a duodenal tube, at least for some time, is the correct treatment, and not seldom saves the patient's life.

One other question now demands consideration. Is the obstruction complete or not? Complete obstruction in the large intestine is evidenced by absolute constipation (after an enema) and rapid distension of the colon.

In the small intestine complete obstruction is indicated by the absence of gas in the evacuated colon and in the coils below the distended loop. If the obstruction is incomplete a second film will demonstrate its re-accumulation after evacuation by an enema.

Having established in this way the fact of obstruction, its probable cause has to be determined. It is manifestly impossible in an article of this kind to consider in detail each variety; this is, moreover, adequately done in all standard textbooks. It is sufficient to say that, having carefully excluded strangulated hernia, and in particular a Richter's hernia, the most probable cause of small 

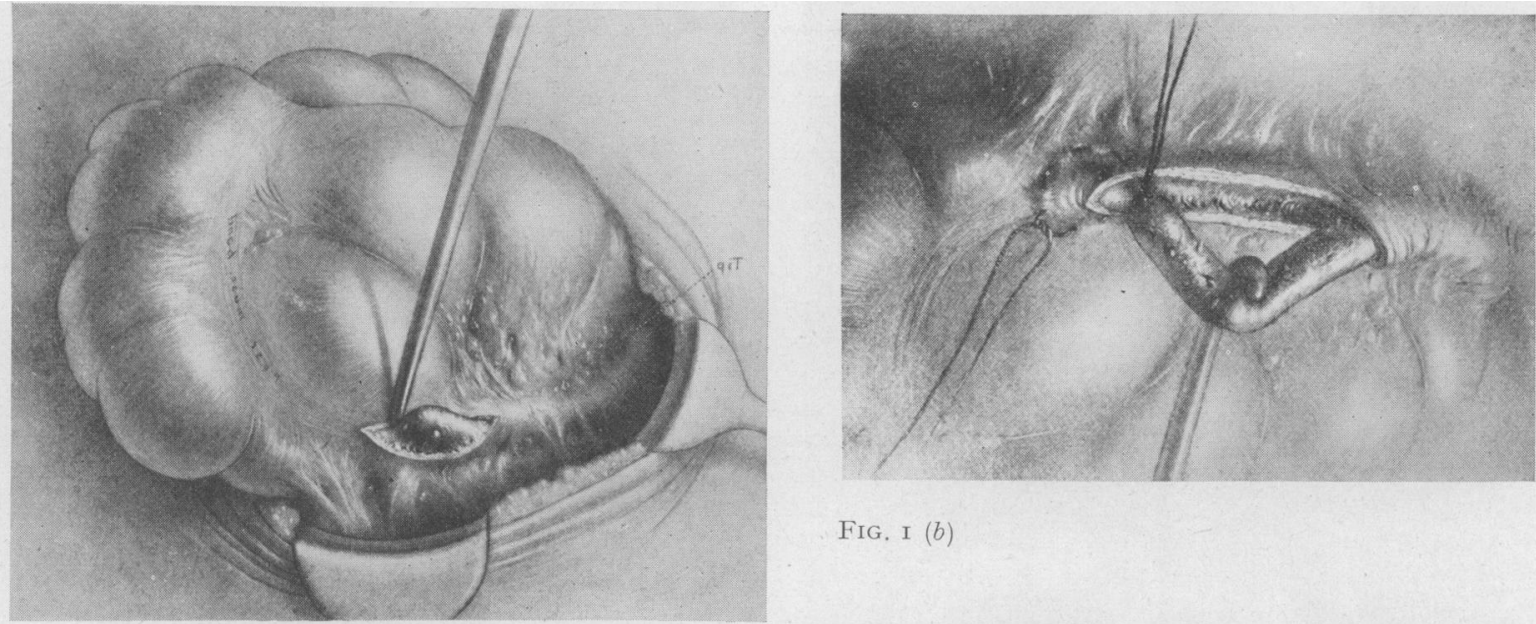

FIG, I (b)

FIG. I $(a)$

FIG. I.- $-(a),(b)$ and $(c)$ illustrating Kelly's method of stripping out the mucosa and submucosa of a densely adherent appendix. (Taken from "Appendicitis," by H. A. Kelly.)

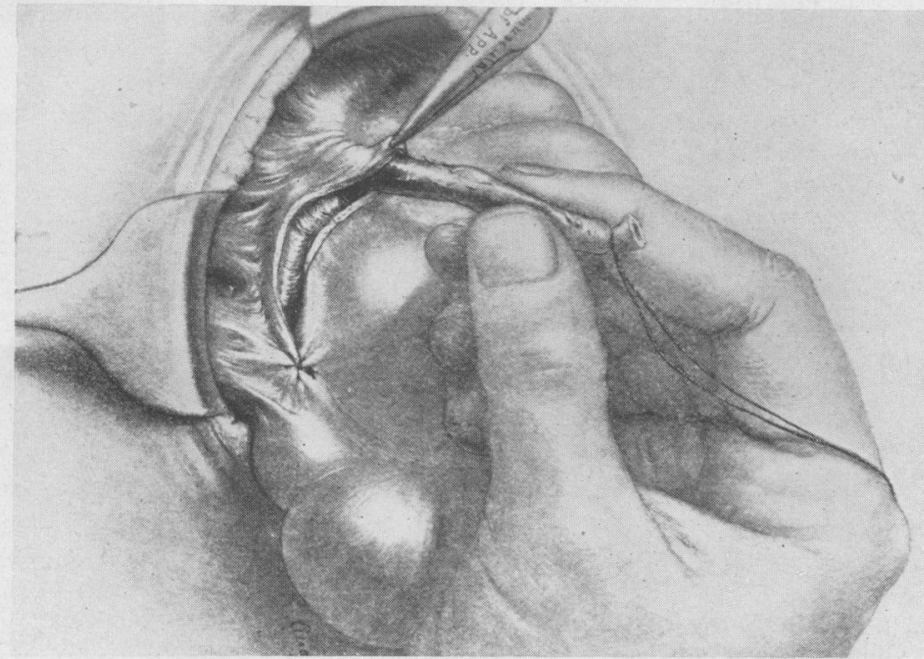

FIG. I $(c)$

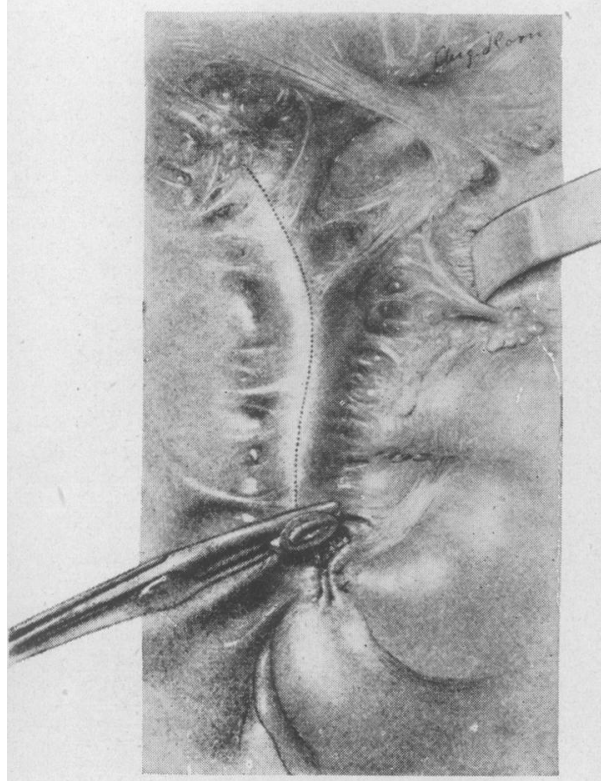

FIG. 2.- $(a)$ and $(b)$ illustrating a nother method of dealing with a densely adherent appendix. The base is divided, the proximal end buried and the appendix slit along its lumen: (Taken from "Appendicitis," by H. A. Kelly.)

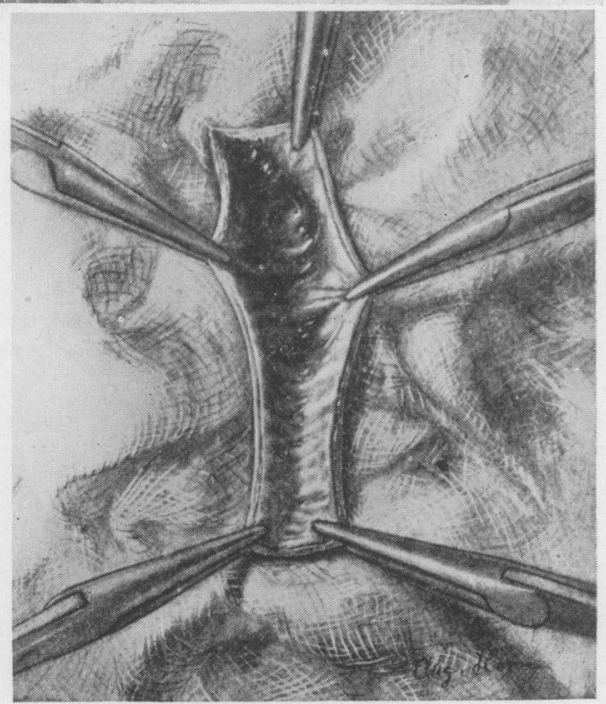

FIG. 2 (b) 

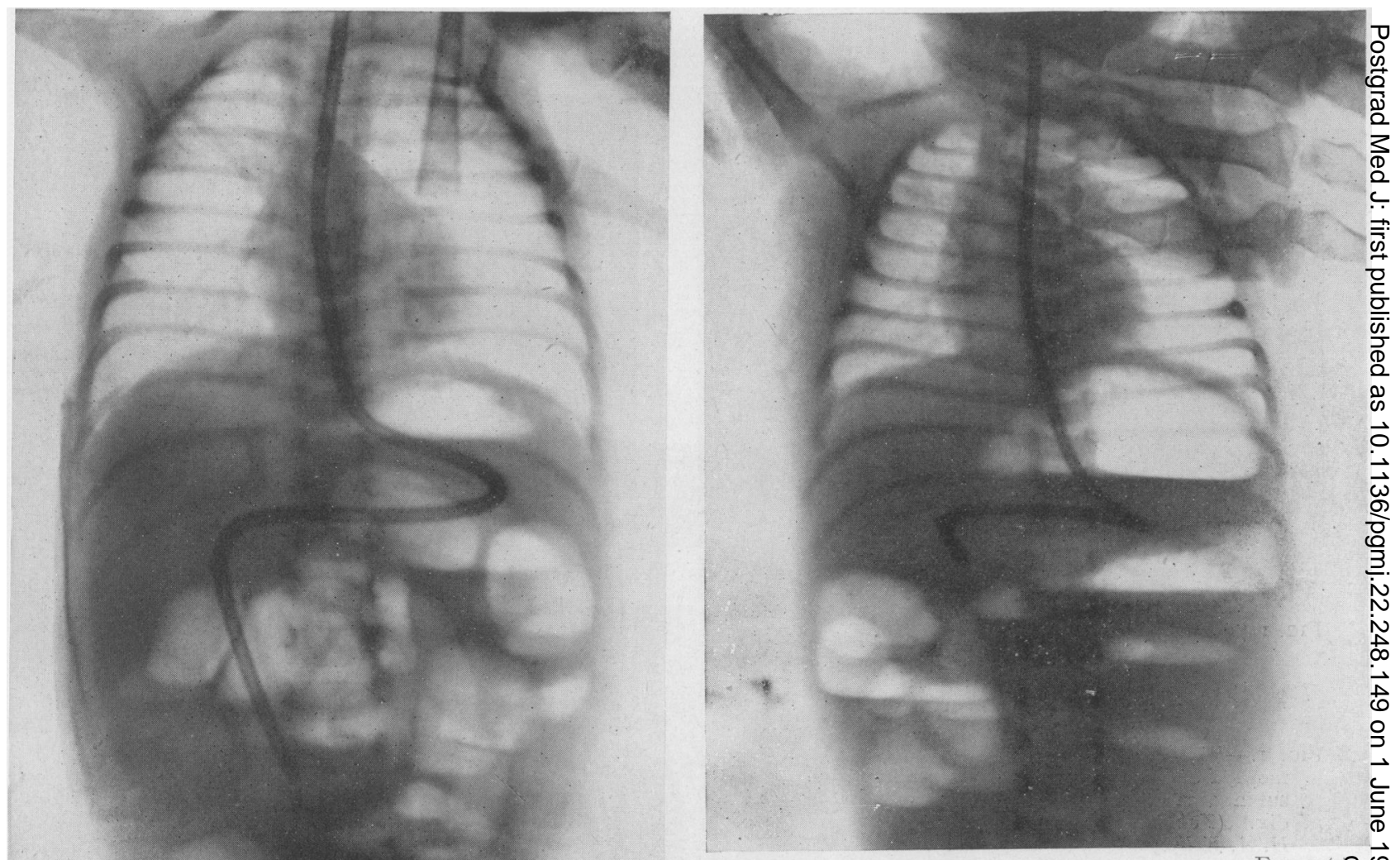

FIG. $3(a)$

FIG. 3.-Plain X-ray of the abdomen of a child with post-operative obstruction of the ilium.

(a) and (b) Antero-posterior and lateral views in the supine position.

(c) Film taken in the upright position showing fluid levels.

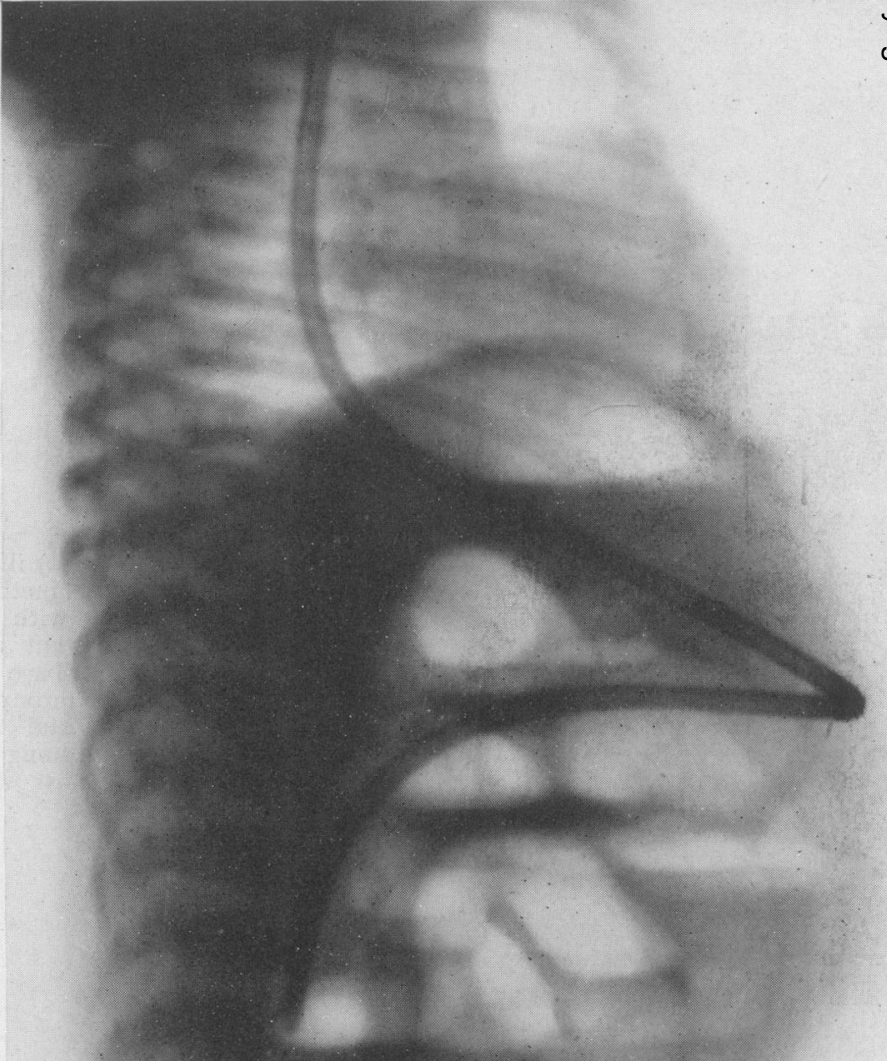


intestinal obstruction is strangulation by bands or adhesions. The history is of great assistance. Symptoms suggestive of tuberculous peritonitis may give an important clue, and the scar of a previous operation is equally significant. A very localised tender spot along or adjacent to the scar points to bowel adherent beneath it, and is a help in planning the incision. Median subumbilical incisions are very prone to produce this kind of aftermath, especially when the operation begins as an exploratory laparotomy and ends as an appendicectomy. Good judgment and experience both indicate that this course should rarely be taken. Apart from a history of an appendicectomy with either drainage or a stormy convalescence, in eight cases out of ten the trouble will be found in the pelvis. Many cases of post-operative adhesions or bands give a history of previous subacute attacks. When no suggestive history is available, a Meckel's diverticulum, a volvulus due to some abnormality of the mesenteric attachments, or an internal hernia naturally come to mind. The latter is rare, sometimes not so acute as other types, and is associated with localised swelling and distension; a soft, rounded fixed tumour may sometimes be felt. Obstruction by a gallstone is apt to be overlooked. A history of cholecystitis and then the onset of progressively severe attacks of colic at decreasingly short intervals are very suggestive. In nine cases out of ten obstruction of the large intestine is due to carcinoma. Volvulus of the sigmoid produces such characteristic distension that it need seldom be missed, and the same may be said of the intussusception of childhood.

In 88 cases of intestinal obstruction the incidence was as follows: strangulated hernia 40 , intussusception 8 , and 40 other varied causes.

\section{Treatment}

In all intestinal obstruction where the integrity of bowel wall is involved, and in large intestinal obstruction where the competence of the ileo-caecal valve converts it into a "closed loop," operation is imperative.

In simple obstructions of the small intestine there is much to be gained by instituting suction through an indwelling duodenal tube. (Fig.6). The distended bowel can be decompressed and alterations in the mechanics may even allow a kink to undo itself. This course is especially indicated in post-operative obstruction due to fresh adhesions or pelvic infective conditions which are best left to subside. Unless obviously necessary it should not be continued for more than about 24 hours, and is safe only if controlled by films taken every three to four hours. Such a procedure has the advantage that the condition can be dealt with "cold" and a clean sweep of involved bowel can be made with a safe end-to-end anastomosis. Obstruction due to plastic lymph and adhesions is very prone to recur. Recently abrased surfaces $c$. are difficult to cover adequately.

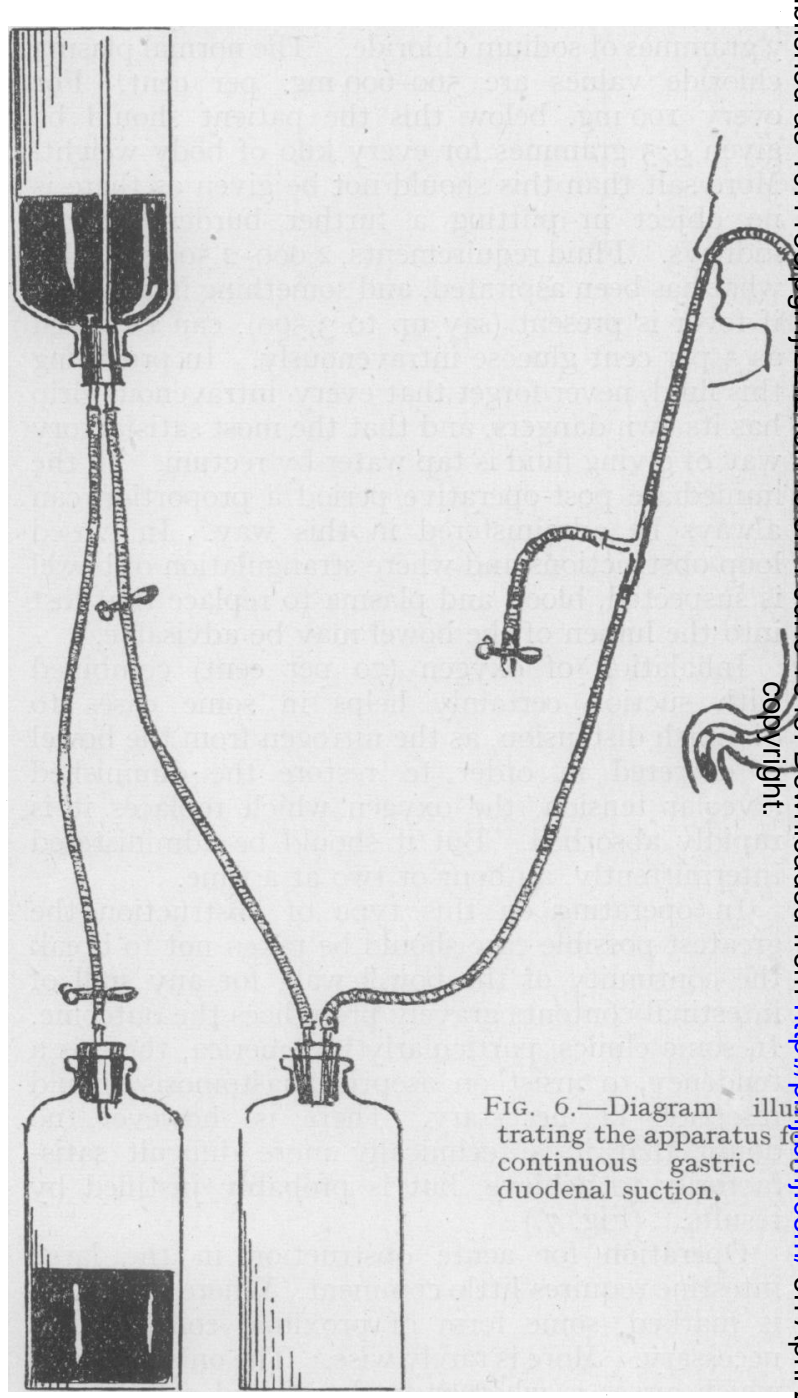

Even when immediate operation is clearly indicated, it is wise first to aspirate the stomach $\underset{\mathrm{W}}{\mathrm{N}}$ and upper jejunum, for many a patient has been ${ }^{\circ}$ drowned in his own regurgitant vomit. Moreover, o most of these patients have some degree of $\stackrel{\mathbb{D}}{\stackrel{2}{ }}$

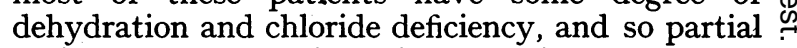
replacement may with advantage be part of the 0 preoperative treatment. The same is also true of large intestinal obstruction as the patients have 
often drunk little or nothing for some days and have lost a considerable amount of fluid into their closed loop. In this connection it must not be forgotten that fluid aspirated and vomited must be replaced parenterally. The best clinical index of fluid requirements is the amount of urine passed, and its chloride content. A patient should pass 700-I,000 c.c. of urine daily, containing at least 3 grammes of sodium chloride. The normal plasma chloride values are $560-600 \mathrm{mg}$. per cent. For every Ioo mg. below this the patient should be given 0.5 grammes for every kilo of body weight. More salt than this should not be given as there is no object in putting a further burden on the kidneys. Fluid requirements, 2,000-2,500 c.c., plus what has been aspirated, and something in addition if fever is present (say up to 3,500), can be given as 5 per cent glucose intravenously. In providing this fluid, never forget that every intravenous drip has its own dangers, and that the most satisfactory way of giving fluid is tap water by rectum. In the immediate post-operative period a proportion can always be administered in this way. In closed loop obstructions and where strangulation of bowel is suspected, blood and plasma to replace that lost into the lumen of the bowel may be advisable.

Inhalation of oxygen (70 per cent) combined with suction certainly helps in some cases to diminish distension, as the nitrogen from the bowel is excreted in order to restore the diminished alveolar tension, the oxygen which replaces it is rapidly absorbed. But it should be administered intermittently, an hour or two at a time.

In operating on this type of obstruction the greatest possible care should be taken not to break the continuity of the bowel wall, for any spill of intestinal contents gravely prejudices the outcome. In some clinics, particularly in America, there is a tendency to insist on aseptic anastomosis should resection be necessary. There is, however, no doubt that it is technically more difficult satisfactorily to achieve, but is probably justified by results. (Fig. 7.)

Operation for acute obstruction in the large intestine requires little comment. Where dilatation is marked, some form of proximal colostomy is necessary. More is rarely wise. The only question which arises is whether to do a blind caecostomy or a transverse colostomy. The former has the disadvantage that it is sometimes followed by serious infection of the abdominal wall. It can, however, be rapidly performed under local anaesthesia, and is equally easily closed. The caecum should be pulled well out and sewn not only to the peritoneum but also to the aponeurosis. The mere insertion of a catheter is most disappointing in its results. A right transverse colostomy under these circumstances should be as simple as possible.
A loop should be pulled through a short incision, the omentum detached, the colon fixed over a glass rod, and the wound closed. Gas can then be evacuated through a wide-bored needle and the bowel opened next day. Never put an intraperitoneal stitch through the wall of a recently obstructed colon, for it is certain that infective material will seep along its track. By the technique suggested no such stitches need be used at all.

\section{(IV) Vascular Occlusions \\ Mesenteric Thrombosis and Embolism}

Acute vascular occlusion of the cardiac coronary vessels with its characteristic symptom, angina pectoris, is so well known that it is almost a synonym for the sudden end to a fruitful middle age. It is the penalty that many a doctor pays for twenty or thirty years of overwork and overstrain. Its counterpart in the abdominal cavity might well be called "angina abdominis," for it is as dramatic, as painful, and as fatal as its better-known elder brother. Fortunately it is probably less common and is amenable to direct surgical attack which, although not invariably successful, has saved many lives; as much as 18 feet of small intestine having been successfully resected on more than one occasion.

In any series of cases large enough to be statistio cally significant, cardiac disease and arterio sclerosis account for 65 per cent of all the cases of arterial occlusion, the one producing embolism and the other thrombosis.

Splenectomy for splenic anaemia, acute abdominal infections, and hepatic disease are mainly responsible for thrombosis of the mesenteric veins. In both cases the result is infarction and gangrene of a varying length of the small intestine. This is caused, not directly by the occlusion itself, but because from it secondary thrombosis spreads to the terminal vascular arches adjacent to the mesenteric border of the bowel, thus preventing the existing rich vascular connections from being effective (Cokinis: Mesenteric Vascular Occlusion, I926).

The inferior mesenteric vessels are also subject to similar occlusions, but the unimpeded anastomosis between its branches often prevents infarction.

Like coronary thrombosis, it is commoner in men than in women in the proportion of $62-38$, but the occasional case occurring in children following acute enteritis must not be forgotten.

The natural history of the disease again resembles coronary thrombosis in that a good many cases 0 have periodical attacks of abdominal pain recurring over a period of weeks or months, and also in that 


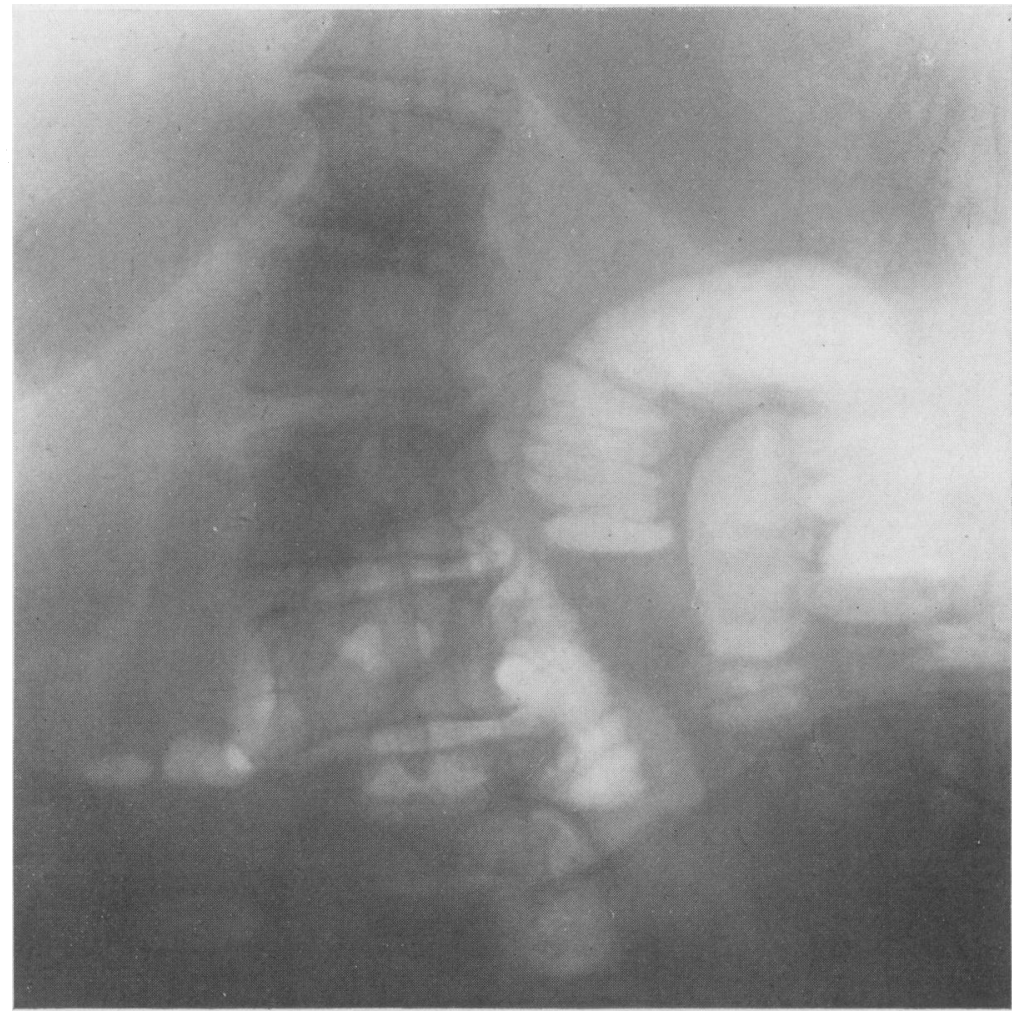

Fig. 4.-Plain X-ray of a patient with intestinal obstruction showing coils of dilated jejunum. The increased number of striations are in marked contrast to the appearance of the ileum shown in Fig. 3. 


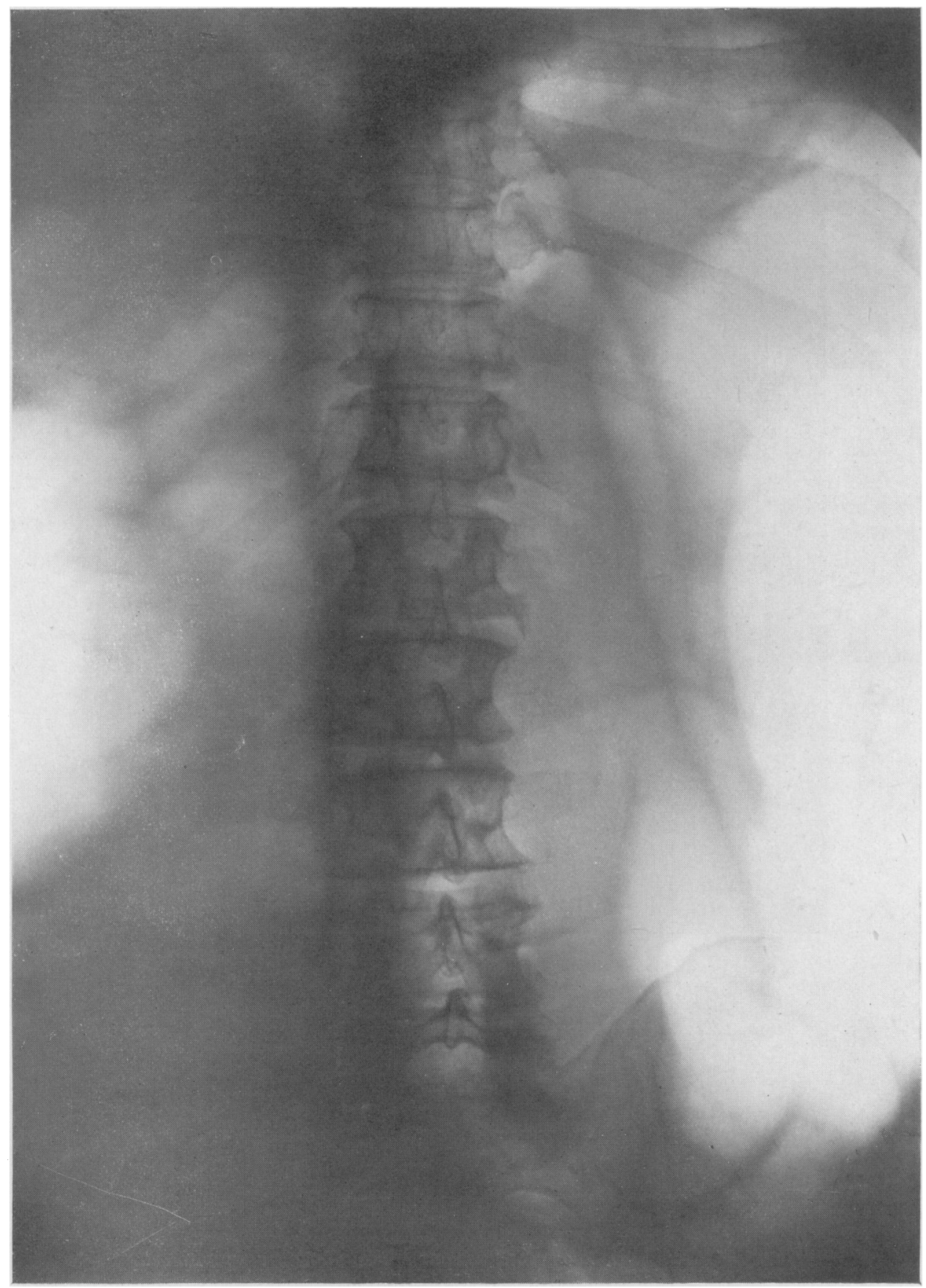

Fig. 5.- Shows the enormous dilation of the sigmoid in a case of volvalus and also dilatation of the caecum, the result of the obstruction. 
there are two clinical types, the fulminating hyperacute case, and the more prolonged type corresponding to a slower spread of the secondary thrombosis. In about half the cases as much as eight days' pain and discomfort may precede the diagnosis of a serious lesion (Jackson, Porter and Quinby, J.A.M.A., 42, I469; 43, 25, 1904). Surgeon Rear-Admiral Sir Gordon Gordon-Taylor has given me permission to quote in full his graphic account of the fulminating attack: "The onset is most dramatic, the anguish intolerable, the shock profound, and the vomiting severe. The pain is experienced all over the abdomen, at first intermittent rather than continuous, but later, during its greatest intensity, the patient draws up his knees and raising the hands in supplication, calls out in agony" (The Essentials of Modern Surgery, Handfield-Jones and Porritt). Indeed the quality of the pain often suggests the correct diagnosis. Pain approaching this in intensity is seen both in acute pancreatitis and in perforated ulcers, but the patients with both these conditions may be in a fit state to walk to hospital; with a mesenteric thrombosis or an embolism, never.

Whether the occlusion is primarily venous or arterial, embolic or thrombolic, the secondary venous thrombosis causes considerable blood loss into the lumen of the bowel. Shock is therefore generally obvious, the patient having a rapid irregular pulse and a subnormal temperature. The extravasated blood may be vomited, but bloody diarrhoea is the general, but not invariable, rule. A vague abdominal tumour due to localised distension is sometimes felt, but when the patient is seen in the later stages the distension is general but not extreme. Visible peristalsis is rare and the same may be said of intestinal colic. Owing to the transudation through the bowel wall, tenderness and "rebound tenderness" are present at some stage of the disease. Later the pain often diminishes or even stops and the clinical picture gradually approaches that of an adynamic ileus, due to general peritonitis. The only treatment likely to be effective is early and wide excision of the involved bowel. In a few cases simple exteriorisation and subsequent anastomosis has been successful. Operation should always be supplemented by transfusion of blood and plasma.

Another condition in which vascular obstruction is a factor of primary importance, is torsion of the pedicle of an ovarian cyst. This occurs more frequently with small cysts, during the early months of pregnancy or the puerperium. It may be precipitated by any strenuous effort. Torsion may take place in either direction and may at first not be complete; frequently it gets untwisted, with a tendency to recurrence. In a few cases there is an associated hyperaemia of the uterine vessels on the same side which may produce some menorrhagia or metrorrhagia. The predominant $\frac{2}{3}$ symptom is pain referred to one side or the whole $\frac{\$}{2}$ lower abdomen. It is severe and intermittent but $\stackrel{\varrho}{c}$ not true colic; it lasts much longer. There is very often a history of milder previous attacks. A considerable amount of free fluid may be poured읃 out, especially if in the early stages there is venous $\frac{\bar{D}}{\bar{N}}$ obstruction leaving the arteries patent; early $\frac{\bar{s}}{\vec{D}}$ complete obstruction produces gangrene and signs $\stackrel{\mathbb{Q}}{\varrho}$ of local peritonitis. There is tenderness if the cyst is out of the true pelvis, and the diagnosis $\vec{A}$ can always be made on pelvic examination. In? cases of doubt examination under anaesthesia is $\vec{\omega}$ desirable and decisive.

Treatment consists in removal of the cyst. $\frac{8}{8}$ Abortion does not usually occur if this is done in the first four months of pregnancy.

\section{Acute Pancreatitis}

Whatever classification of abdominal calamities is $\vec{a}$ adopted, acute pancreatitis remains in a class by $\overrightarrow{0}$ itself because, although vascular changes may con-음 stantly dominate the clinical picture, infection by $\overrightarrow{-}$ one or other of the possible routes is a necessary $\subseteq$ concomitant factor. Haemorrhage unaccompanied by infection does not cause acute pancreatitis. It $\overrightarrow{0}$ is possible that either haemorrhage or infectiog $\frac{\phi}{\sigma}$ may be the primary lesion. In the former case the superadded infection disintegrates the gland ar increases the haemorrhage, and in the latter it causes the haemorrhage which increases the disintegration of the gland. Infection may come from $\stackrel{\circ}{\circ}$ the blood stream, from the gallbladder and bile $\stackrel{\Phi}{\varrho}$ ducts via the lymphatics, or directly along the $\overrightarrow{\overrightarrow{0}}$ pancreatic duct when the ampulla is blocked by a 3 stone. The blood stream is certainly an occasional source, since the disease occurs in children, in infective endocarditis, and in typhoid, but the most significant pathological fact is that the gallbladder contains stones in over 80 per cent of all the cases, and in 60 per cent they are also present in the common bile duct. In less than 20 per cent $\delta$ are they impacted in the ampulla. When these pathological data are related to the intimate and 을 rich anatomical connections between the lymphatics $D$ of the bile tract and the head of the pancreas behind the peritoneum, it is difficult to avoid the conclusion $\bar{N}$ that the lymphatics are the most probable pathway for infection to travel from the gallbladder to the $\tilde{N}$ pancreas. Regurgitation of the infected bile suffi- N cient to cause pancreatitis is only a convincing 0 explanation in those cases which have stoneso impacted in the ampulla of Vater. However, initiated, there follows a complex cause-effect $\stackrel{\infty}{+}$ chain of events-infection, haemorrhage, disinte- $\square$ gration, the setting free of trypsin and its activation by colon bacilli, blood and serum. This may 
produce anything from a massive haemorrhage to the oedema of the pancreatic head so often seen when operating for gallstones. Herein lies the explanation of the diverse clinical accounts given of acute pancreatitis. There may be a fulminating haemorrhagic type, which is rapidly fatal, a tense generalised white oedema which subsides, or necrosis and abscess formation which needs drainage.

Moynihan's classic description based on his own experience and the work of Fitz in I899 is familiar to everyone. Time and experience have brought changes only in perspective, and these hyperacute cases must now be seen against a background of many others not nearly so severe, which subside naturally and are often imprecisely diagnosed.

There is frequently a history suggestive of cholelithiasis and sometimes a slight tinge of jaundice, and there may be a few days or hours of intermittent upper abdominal pain varying in severity, but the onset of the main attack is apt to be catastrophic. The pain is agonising, causing shock and prostration. The patient first writhes in bed and thereafter lies still, cold, sweating, and motionless, with a pulse rapidly mounting to 120-160 per minute. The pain is generalised in the region above the umbilicus, and in the less severe cases, to the right hypochondrium. It is not relieved by morphia and is rhythmic in quality, perhaps corresponding to the fresh haemorrhages which occur from time to time. Characteristically it radiates to the back and left shoulder, and after some hours, subsides to become a heavy, dull, unvarying ache in the epigastrium and the back. With this goes the most resistant rigidity, a tenderness which makes any attempt at deep palpation merely fruitless torture. This rigidity tends to remain localised in the upper abdomen, certainly does not spread downwards with the same rapidity and certainty as does the peritonitis caused by a perforated ulcer. The early rise in pulse rate, too, is in marked contrast, so is the continuous retching and vomiting of small amounts of fluid, probably due to the accumulation of toxic irritating fluid in the lesser sac and round the coeliac ganglia. Milder cases do not present either this marked unvarying rigidity or persistent vomiting, probably because the changes are more limited; a vague mass corresponding to a distended lesser sac or the pancreas itself may be felt.

Another characteristic of acute pancreatitis often described is a blotchy cyanosis, generalised, but often most marked around the umbilicus. This, however, is seen in other conditions and was most striking in a case seen recently with a perforation of an acute lower oesophageal ulcer. Patchy haemorrhagic areas around the umbilicus and in the loin have been noticed by Cullen and Grey Turner.
In the first twelve hours of an attack there is nearly always a rise in the urinary diastase, the normal being 20 units, this may be increased to between 200 and 600 units. The blood diastase is probably more reliable but is less often done. These two tests are in practice valuable because all descriptions of acute pancreatitis tend to oversimplify the clinical picture, and the diagnosis at the bedside is often really difficult.

\section{Treatment}

There is a tendency nowadays to adopt a more conservative attitude to treatment, which comes with the realisation that many of the less acute cases will settle down, though perhaps later it may be necessary to drain the abscess or remove a slough, either through the loin or the lesser sac. Even in the hyperacute cases it is hard to see what surgery has to offer beyond the evacuation of bloodstained exudate from the small omental bursa, and unless localised abscess formation is present nothing more than this should be done. In spite of the fact that surgeons are nowadays "acute pancreatitis conscious," laparotomy is quite often performed because of the imperative necessity for diagnostic certainty. When a rapid exploration of the stomach and gallbladder has proved negative, the lesser sac should be explored, either from above or below the stomach, and the pancreaso examined. Sometimes fat necrosis gives the diagnosis away as soon as the abdomen is opened. In such cases, before closing the abdomen, a drainage tube should be placed down to the pancreas. If the gallbladder or common bile ducts contain stones, the gallbladder should be simply drained, and the whole condition dealt with later, when time for the precision necessary for exposure of the ducts is available. The complete abdominal rest which can be obtained by suction through a Ryle's tube, morphia, and the intravenous administration of blood, plasma, and glucose saline are the most important and do more than anything else to lead to subsidence or localisation.

In an article of this kind, where the author has of necessity to adopt the role of a "surgical columnist," oversimplification, improper emphasis, and apparent dogmatism are as unavoidable as they are obvious. These faults are justified only if they lead an observer to see acute abdominal lesions and their protean manifestations as part of a biological process rather than as isolated events in clinical experience. Regarded in this way they always retain their fascination, as each successive case continues to call for the best judgment of even the most experienced surgeon.

For the statistical material contained in this article I have to thank my registrar, Mr. Eric 


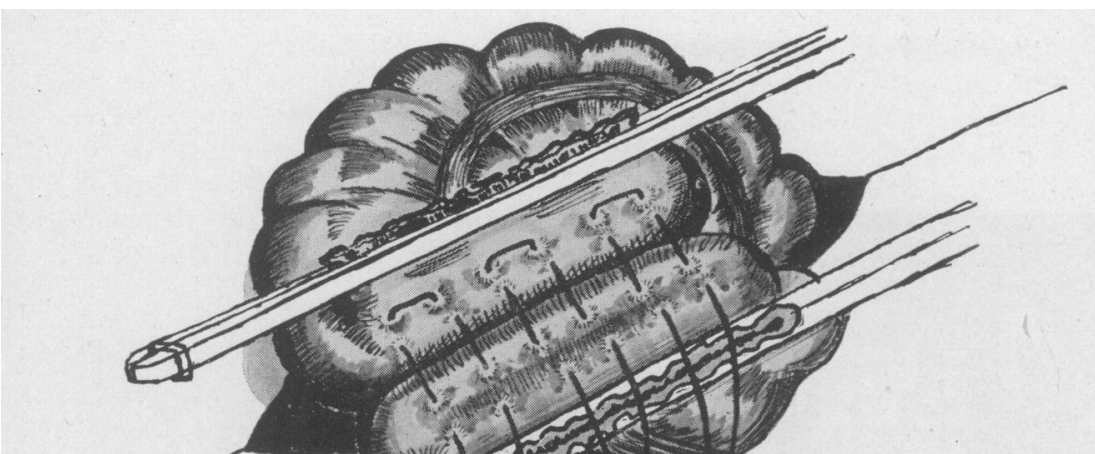

Fig. 7.-Drawings illustrating our صి method of performing aseptic $\overline{\mathbf{C}}$ anastomosis of the large intestine. $\vec{D}$ For the'small intestine the clamps are placed obliquely to conserve. the blood supply of the anti- $\overrightarrow{\overline{\vec{n}}}$ mesenteric border and increase the size of the lumen.

FIG. 7A.-A posterior view of mattress sutures has been placed $\vec{\omega}$ ready to be tied after three or four have been inserted.

FIG. 7в.-A further posterior layer consisting of a continuous "basting" stitch, each end held loosely by artery forceps.
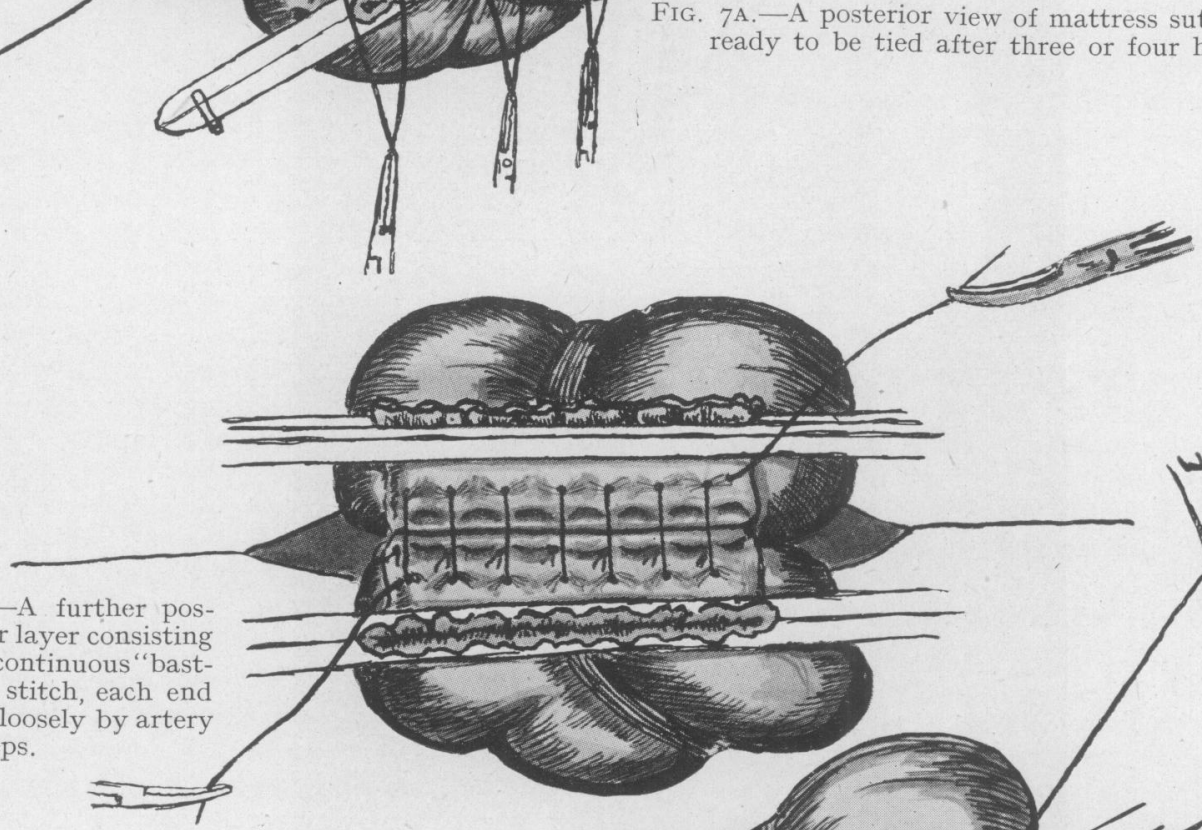

T1
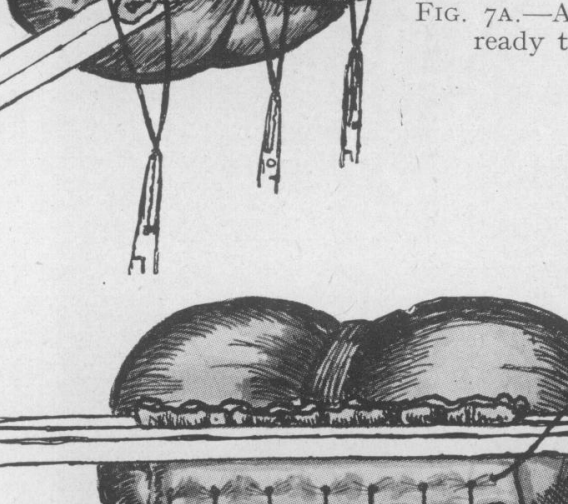

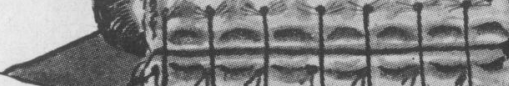

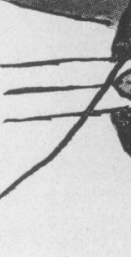

a)
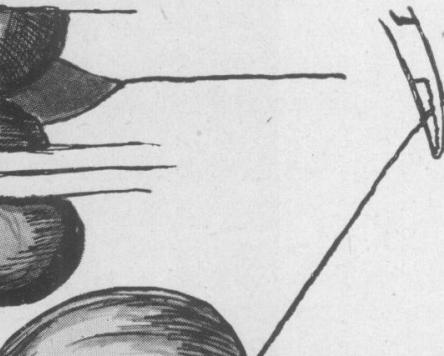

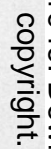

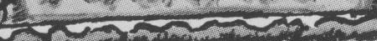

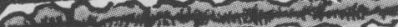
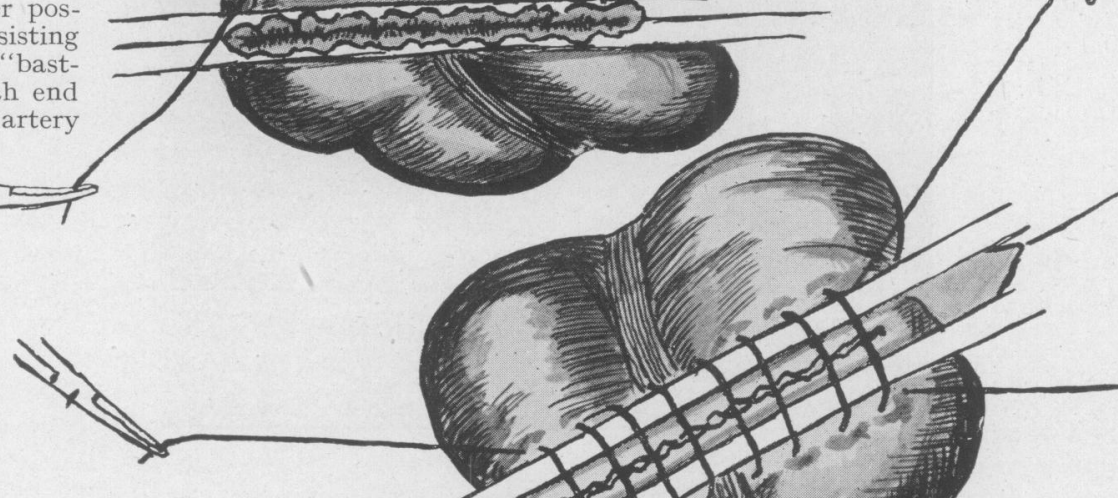


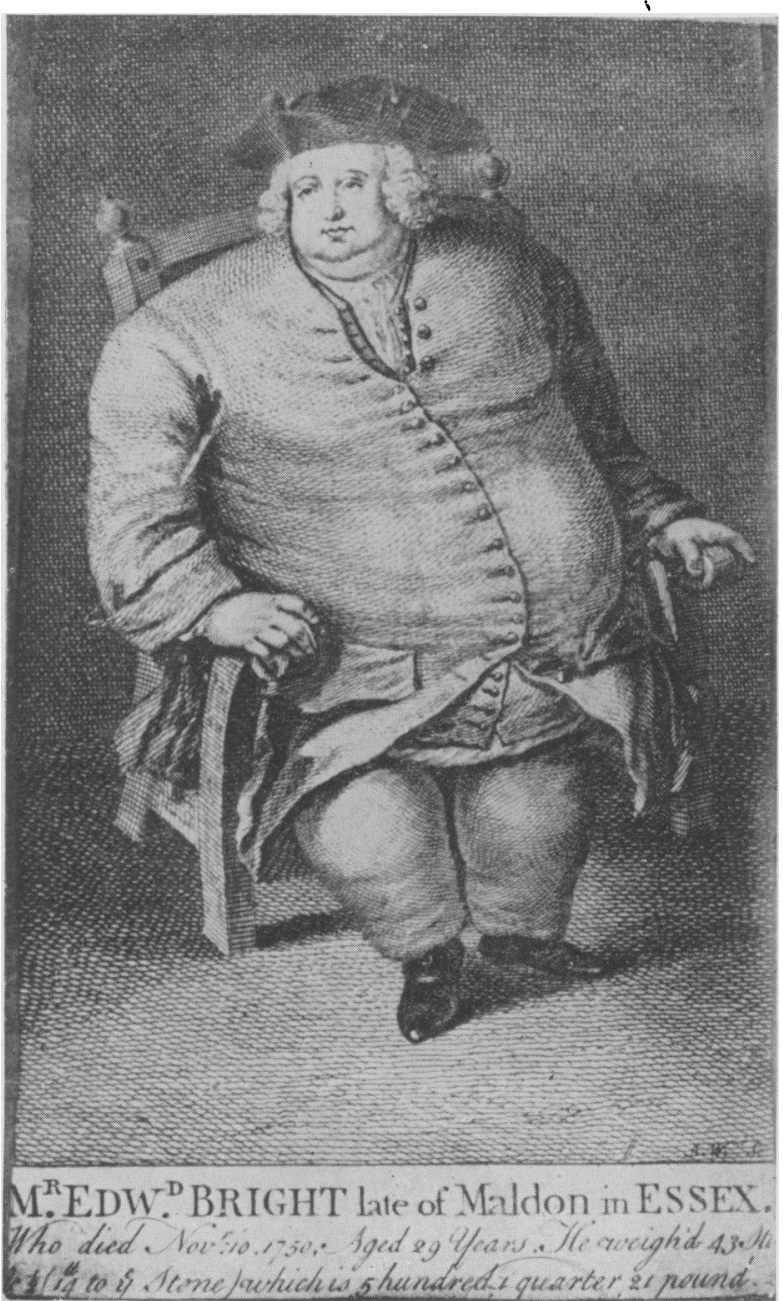

Mr. Edward Bright of Maldon, who died November 10, I750, aged 29 years. His height was $5 \mathrm{ft}$. $9 \frac{1}{2}$ ins., chest 66 ins., waist 83 ins., around the arm 26 ins., and around the leg 32 ins. His weight was 44 'stone, or $6 \mathrm{r} 6 \mathrm{lbs}$. net. After his death, to decide a wager, seven men were buttoned up in his waistcoat. 
Gardiner, and both he and many other senior assistants are responsible for much of what I have learnt and for a large part of whatever success I have had in treating patients who are critically ill.

My friend and colleague Mr. Albert Davis, has always put his knowledge and experience at my disposal and any merit which the part of this article dealing with pelvic surgery may have are due to his guidance; the faults are mine.
I have to thank Mr. Mortimer Woolf for permission to reproduce Fig. 5 and Dr. Leonard $\varrho$ Symonds for the drawings in Figs. 6 and 7 .

Finally, it is a pleasure to thank Sir Gordon $\stackrel{2}{2}$ Gordon-Taylor for permission to quote his writings. $\stackrel{c}{.}$ His experience is a sort of National Trust for his $\overrightarrow{\vec{F}}$ colleagues, and this is a welcome opportunity to acknowledge much wise advice given freely over $\frac{\mathrm{C}}{0}$ many years.

\section{ADIPOSITY}

By Raymond Greene, M.A., D.M., M.R.C.P. (Hon. Physician Metropolitan Hospital)

\section{The Importance of Adiposity*}

In the words of Donald Hunter (I933) "the victims of obesity are . . . to be pitied for many reasons. They must of necessity suffer from a restriction of physical activity, they have a tendency to develop flat-feet and arthritis, they also have an unpleasant tendency to excessive sweating, and they are difficult to anaesthetise, operate on, and nurse. Moreover, they are predisposed to diabetes, bronchitis, and possibly to hyperpiesia." It may be added that obesity is a cause of great unhappiness to many women. Though a lucky few are prone to laugh and grow fat, the majority are sufficiently conscious of the preference for slimness of the ordinary man to become self-conscious and self-critical, to lose their self-confidence and sometimes to sink into a state of severe depression. The importance of adiposity is well shown by the analysis by Dublin and Lotka (I936) of the life histories of I92,304 men accepted for life insurance.

\section{Table I}

Influence of Weight on Mortality. Deaths per I00,000

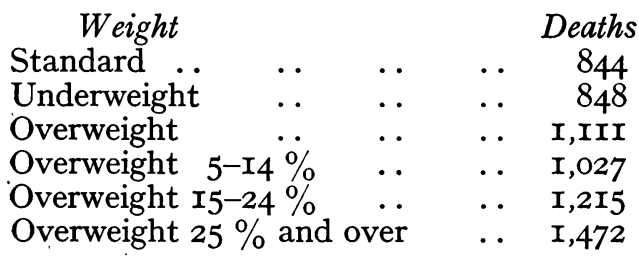

When the age of the "life" is considered, the influence of adiposity becomes still more obvious.

* Adiposity is derived from the Latin adeps (fat), obesity from the Latin $o b$ and esus (having eaten). The former is more generally accurate.
Table 2

Influence of Weight on Mortality. Effect of age, Deaths per 100,000

\begin{tabular}{llccc}
\multicolumn{1}{c}{ Weight } & \multicolumn{3}{c}{ Age } \\
& \multicolumn{3}{c}{ Under } & Over 45 \\
Standard . & $\ldots$ & 463 & I,308 \\
Underweight & $\ldots$ & 498 & I,274 \\
Overweight & $\ldots$ & 527 & I,824
\end{tabular}

Table 3

Influence of Overweight on Mortality in Persons age 45 to 50

$\begin{array}{cc}\text { Pounds Overweight } & \text { over Average } \\ \text { Io } & 8 \\ 20 & 18 \\ 30 & 28 \\ 40 & 45 \\ 50 & 56 \\ 60 & 67 \\ 70 & 8 \mathrm{I} \\ 90 & \mathrm{II} 6\end{array}$

In other words, a mere stone of extra weight in middle age, lessens a man's expectation of life by about Io per cent, and "fifty pounds overweight at age 45 imposes as much extra mortality as valvular 은 heart disease" (Fisk, quoted by Newburgh, I942).

\section{The Definition of Adiposity}

The decision as to whether a patient is too fat $N$ or not must obviously be largely a question of $N$ taste. Many Oriental races prefer fat women and $\omega$ even in one country fashions change. We must fall back upon averages considered in the light of common sense. The most reliable averages for children are $\stackrel{\Phi}{\oplus}$ probably those of the Child Health Association and for adults those of Fisk (I923). Anderson's Nomogram is very convenient in practice. 